\title{
A Modification of Differential Transform Method for Solving Systems of Second Order Ordinary Differential Equations
}

\author{
S. Al-Ahmad ${ }^{1}$, I. M. Sulaiman ${ }^{1, *}$, M. Mamat ${ }^{1}$, L. G. Puspa ${ }^{2}$ \\ ${ }^{1}$ Faculty of Informatics and Computing, Universiti Sultan Zainal Abidin, Malaysia \\ ${ }^{2}$ Faculty of Business and Management, Sultan Zainal Abidin University, Malaysia
}

Received May 19, 2020; Revised July 2, 2020; Accepted July 20, 2020

Copyright $\subseteq 2020$ by authors, all rights reserved. Authors agree that this article remains permanently open access under the terms of the Creative Commons Attribution License 4.0 International License

\begin{abstract}
The method of differential transform (DTM) is among the famous mathematical approaches for obtaining the differential equations solutions. This is due to its simplicity and efficient numerical performance. However, the major drawback of the DTM is obtaining a truncated series solution which is often a good approximation to the true solution of the equation in a specified region. In this study, a modification of DMT scheme known as MDTM is proposed for obtaining an accurate approximation of ordinary differential equations of second order. The scheme whose procedure is designed via DTM, the Laplace transforms and finally Padé approximation, gives a good approximate for the true solution of the equations in a large region. The proposed approach would be able to overcome the difficulty encountered using the classical DTM, and thus, can serve as an alternative approach for obtaining the solutions of these problems. Preliminary results are presented based on some examples which illustrate the strength and application of the defined scheme. Also, all the obtained results corresponded to exact solutions.
\end{abstract}

Keywords Differential Transform Method, Systems of Second Order ODEs, Padé Approximants, Laplace Transform

\section{Introduction}

Consider the $n$ order system of ODEs

$$
\left\{\begin{array}{c}
\varphi_{1}\left(x, y_{1}(x), y_{1}{ }^{\prime}(x) \ldots, y_{1}{ }^{(n)}(x), y_{2}(x), y_{2}{ }^{\prime}(x) \ldots, y_{2}{ }^{(n)}(x), y_{N}(x), y_{N}{ }^{\prime}(x) \ldots, y_{N}{ }^{(n)}(x)\right)=0 \\
\varphi_{2}\left(x, y_{1}(x), y_{1}{ }^{\prime}(x) \ldots, y_{1}{ }^{(n)}(x), y_{2}(x), y_{2}{ }^{\prime}(x) \ldots, y_{2}{ }^{(n)}(x), y_{N}(x), y_{N}{ }^{\prime}(x) \ldots, y_{N}{ }^{(n)}(x)\right)=0 \\
\vdots \\
\varphi_{N}\left(x, y_{1}(x), y_{1}{ }^{\prime}(x) \ldots, y_{1}{ }^{(n)}(x), y_{2}(x), y_{2}{ }^{\prime}(x) \ldots, y_{2}{ }^{(n)}(x), y_{N}(x), y_{N}{ }^{\prime}(x) \ldots, y_{N}{ }^{(n)}(x)\right)=0
\end{array}\right.
$$

where $\varphi_{i}$ for $i=1,2,3, \ldots, N$ are nonlinear continuous functions of its argument [1]. The above system has been studied by numerous scientists and engineers due to its importance in vast applications including mathematical and physical models. Recently, much attention had been given to numerical solutions of these systems of ODEs. One of the efficient numerical methods for solving this system is the Differential transform method (DTM).

The DTM which constructs a polynomial analytical solution was first introduced by Zhou [3] as a new idea for solving differential equations (DEs). This form of polynomials is used as the approximate to exact solutions. The idea of DTM is based on the concept of Taylor's series [4-6], whose solution is usually obtained in series form. DTM is a reiterative approach for obtaining solutions of Taylor series DEs. Recently, the DTM has been extended to boundary value problems, difference equations, initial value problems.

However, the DTM has some drawbacks as stated in the abstracts [7]. To overcome this drawback, the study proposed an alternative scheme to improve the efficiency of DTM by modifying the series solution of systems of second order ODEs. The proposed scheme starts with the DMT obtained truncated series by applying the Laplace transform, followed by employing the Padé approximants to transform series into a meromorphic function, and lastly obtaining an analytic solution using the inverse Laplace transform. This obtained solution may be an improved approximation or periodic solution compared to the DTM truncated series solution. The proposed MDTM will be used without requiring any linearization or smallness assumptions [2].

The rest of the paper is arranged as follows. In this paper: Section 2 discusses brief overview and some fundamental results of DTM, Padé approximants and Laplace transform. The proposed scheme is applied to 
various problems in order to illustrate its simplicity and effectiveness in Section 3. Lastly, the conclusion and discussion are in Section 4.

\section{Preliminaries}

This section presents some definitions of DTM and Padé approximants.

\subsection{Differential Transform Method}

\section{Definition 2.1.[8]}

For a function $f(x)$ that is analytical at $x_{0}$ in the domain of interest, then

$$
F(k)=\frac{f^{(k)}\left(x_{0}\right)}{k !} .
$$

whose inverse differential transform is

$$
f(x)=\sum_{k=0}^{\infty} F(k)\left(x-x_{0}\right)^{k} .
$$

Let the DEs of $u(x), g(x)$, and $h(x)$ be $U(k), G(k)$, and $H(k)$ respectively at $x_{0}=0$. Then the known DTM operations are presented in Table 1.

Table 1. Differential Transform

\begin{tabular}{|c|c|}
\hline Original function & Transformed function \\
\hline$u(x)=g(x)+h(x)$ & $U(k)=G(k)+H(k)$ \\
\hline$u(x)=c g(x)$ & $U(k)=c G(k)$ \\
\hline$u(x)=\frac{d^{n} g(x)}{d x^{n}}$ & $U(k)=\frac{(k+n) !}{k !} G(k+n)$ \\
\hline$u(x)=g(x) h(x)$ & $U(k)=\sum_{i=1}^{k} G(i) H(k-i)$ \\
\hline$u(x)=x^{n}$ & $U(k)=\delta(k-n)$ \\
\hline$u(x)=\exp (c x)$ & $U(k)=\frac{c^{k}}{k !}$ \\
\hline$u(x)=\cos (\omega x)$ & $U(k)=\frac{\omega^{k}}{k !} \cos \left(\frac{k \pi}{2}\right)$ \\
\hline$u(x)=\sin (\omega x)$ & $U(k)=\frac{\omega^{k}}{k !} \sin \left(\frac{k \pi}{2}\right)$ \\
\hline
\end{tabular}

Theorem 2.1 [9]. If $f(y)=y^{m}$, then

$$
F(k)=\left\{\begin{array}{c}
(Y(0))^{m}, \quad k=0 \\
\frac{1}{Y(0)} \sum_{r=1}^{k}\left(\frac{(m+1) r-k}{k}\right) Y(r) F(k-r), k \geq 1
\end{array}\right.
$$

The proof is obvious from the operations defined in Table 1 and definitions (2.1) - (2.2).

A differential equation can be transformed into an equation of algebraic form in the $K$-domain using differential transform and the Taylor series expansion finite-term to obtain $f(t)$ as

$$
f(t)=\sum_{k=0}^{N} F(k) \frac{\left(t-t_{0}\right)^{k}}{k !}+R_{N+1}(t)
$$

The series solution (2.3) possesses a rapid convergence rate but only in a small region. However, the convergence is often very slow in the wide region, and thus, yielding an inaccurate truncated result.

In the MDTS, we start by applying the Laplace transform to the obtained DMT truncated series, followed by employing the Padé approximants to transform the obtained series into a meromorphic function, and lastly obtaining an analytic solution using the inverse Laplace transform. This obtained solution may be an improved approximation or periodic solution compared to the DTM truncated series solution. For further reference on differential transform and DTM see [8-13, 18].

\subsection{Padé Approximation [14, 15]}

The approximants of Padé refers to the ratio of two polynomials derived from Taylor series expansion coefficients function $y(x)$. The $[L / M]$ Padé approximations to $y(x)$ are defined by

$$
\left[\frac{L}{M}\right]=\frac{P_{L}(x)}{Q_{M}(x)}
$$

and $P_{L}(x)$ and $Q_{M}(x)$ are known as the polynomials of degree at most $L$ and $M$ respectively. More so,

$$
\begin{gathered}
y(x)=\sum_{i=1}^{\infty} a_{i} x^{i}, \\
y(x)-\frac{P_{L}(x)}{Q_{M}(x)}=\mathrm{O}\left(x^{L+M+1}\right)
\end{gathered}
$$

are the formal power series determining the coefficients of the polynomials $P_{L}(x)$ and $Q_{M}(x)$ by the equation.

It is obvious the numerator and denominator of $[L / M]$ remain unchanged even after multiplying by a constant, thus, the following normalization condition is imposed

$$
Q_{M}(0)=1 \text {. }
$$

Lastly, $P_{L}(x)$ and $Q_{M}(x)$ are required to have no common factors. Rewriting $P_{L}(x)$ and $Q_{M}(x)$ coefficient as

$$
\left\{\begin{array}{c}
P_{L}(x)=p_{0}+p_{1} x+p_{2} x^{2}+\cdots+p_{L} x^{L} \\
Q_{M}(x)=q_{0}+q_{1} x+q_{2} x^{2}+\cdots+q_{M} x^{M}
\end{array}\right.
$$

also, from (2.7) and (2.8), we can multiply (2.6) by $Q_{M}(x)$, thereby linearizing the coefficient equations.

Rewriting (2.6) in more detailed form, gives

$$
\begin{gathered}
\left\{\begin{array}{c}
a_{L+1}+a_{L} q_{1}+\cdots+a_{L-M+1} q_{M}=0 \\
a_{L+2}+a_{L+1} q_{1}+\cdots+a_{L-M+2} q_{M}=0 \\
\vdots \\
a_{L+M}+a_{L+M-1} q_{1}+\cdots+a_{L} q_{M}=0
\end{array}\right. \\
\left\{\begin{array}{c}
a_{0}=p_{0} \\
a_{0}+a_{0} q_{1}=p_{1} \\
a_{2}+a_{1} q_{1}+a_{0} q_{2}=p_{2} \\
\vdots \\
a_{L}+a_{L-1} q_{1}+\cdots+a_{0} q_{L}=p_{L}
\end{array}\right.
\end{gathered}
$$

To obtain the solutions of the above equations, we begin with all the unknown of the linear equations (2.9) 
$q$ 's. Once we obtain the values of the $q$ 's, then (2.10) will produce a formula for-all the defined unknown $p$ 's, and thus, concludes the solution.

If equation (2.9) and equation (2.10) are non-singular, then, the solution can be obtained directly as presented in equation (2.11) [15], which implied that (2.11) holds, but if the index of the lower sum exceeds the upper, then, it would be substituted by zero:

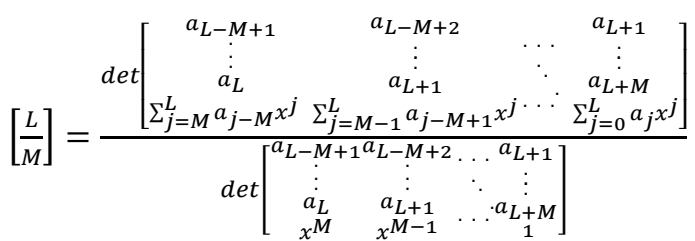

From this step, the symbolic calculus software, MATLAB is often employed to obtain the Padé approximants diagonal matrix of orders including $[2 / 2],[4 / 4]$ or $[6 / 6]$.

\section{Remark}

Note that typically the Padé approximant, obtained from a partial Taylor sum, is more accurate than the latter. However; the Padé, being a rational expression, has poles, which are not present in the original function. It is a simple algebraic task to expand the form of an $[N, M]$ Padé in a Taylor series and compute the Padé coefficients by matching with the above [14].

\section{Numerical Results}

This section presents the solutions of systems of second order ODEs by reducing higher order DE's to system of first order DEs.

Problem 3.1: Consider the following second order systems of differential equations [1]

$$
\left\{\begin{array}{c}
y_{1}^{\prime \prime}(t)+y_{1}(t)-y_{2}{ }^{\prime \prime}(t)-4 y_{2}(t)=0 \\
y_{1}^{\prime}(t)+y_{2}^{\prime}(t)=\cos (t)+2 \cos (2 t)
\end{array},\right.
$$

subject to

$$
\begin{cases}y_{1}(0)=0, & y_{1}{ }^{\prime}(0)=1 \\ y_{2}(0)=0, & y_{2}{ }^{\prime}(0)=2\end{cases}
$$

and exact solution

$$
\left\{\begin{array}{c}
y_{1}(t)=\sin (t) \\
y_{2}(t)=\sin (2 t)
\end{array} .\right.
$$

Transforming Eq. (3.1) with Eq. (3.2), to obtain $\left\{\begin{array}{c}(k+1)(k+2) Y_{1}(k+2)+Y_{1}(k)-(k+1)(k+2) Y_{1}(k+2)-4 Y_{2}(k)=0 \\ (k+1) Y_{1}(k+1)+(k+1) Y_{2}(k+1)=\frac{1}{k !} \cos \left(\frac{k \pi}{2}\right)+\frac{2^{(k+1)}}{k !} \cos \left(\frac{k \pi}{2}\right)\end{array}\right.$ and

$$
\left\{\begin{array}{l}
Y_{1}(0)=0, Y_{1}(1)=1 \\
Y_{2}(0)=0, Y_{2}(1)=2
\end{array}\right.
$$

Replacing (3.4) in (3.3), yields the values of $Y_{1}(k)$ and $Y_{2}(k)$ presented in Table 2:

Table 2. The values of $Y_{1}(k)$ and $Y_{2}(k)$ for Problem 3.1

\begin{tabular}{|c|c|c|c|c|c|c|}
\hline$k$ & 0 & 1 & 2 & 3 & 4 & 5 \\
\hline$Y_{1}(k)$ & 0 & 1 & 0 & -0.1666666667 & 0 & 0.0083333333 \\
\hline$Y_{2}(k)$ & 0 & 2 & 0 & -1.3333333333 & 0 & 0.2666666667 \\
\hline
\end{tabular}

Using the rule in (2.2), we obtain an approximate solution of equation (3.1) as follows

$$
\left\{\begin{array}{l}
y_{1}(t)=t-0.1666666667 t^{3}+0.0083333333 t^{5}+\cdots \\
y_{2}(t)=2 t-1.3333333333 t^{3}+0.2666666667 t^{5}+\cdots
\end{array}\right.
$$

To improve the efficiency of the solution of differential transform (3.5), and illustrate the efficiency of the MDTS, we proceed as follows. By taking just $\mathcal{O}\left(t^{5}\right)$ terms from (3.5), the MDTS is executed as follows:

Applying the Laplace transform to the first $\mathcal{O}\left(t^{5}\right)$ terms from (3.5), yields

$$
\left\{\begin{array}{l}
\mathcal{L}\left(y_{1}(t)\right)=\frac{1}{s^{2}}-\frac{1}{s^{4}} \\
\mathcal{L}\left(y_{2}(t)\right)=\frac{2}{s^{2}}-\frac{8}{s^{4}}
\end{array}\right.
$$

For simplicity, let $s=\frac{1}{z}$; then

$$
\left\{\begin{array}{c}
\mathcal{L}\left(y_{1}(t)\right)=z^{2}-z^{4} \\
\mathcal{L}\left(y_{2}(t)\right)=2 z^{2}-8 z^{4}
\end{array} .\right.
$$

The Padé approximants $\left[\frac{2}{2}\right]$ gives

$$
\left[\frac{2}{2}\right]=\left\{\begin{array}{c}
\mathcal{L}\left(y_{1}(t)\right)=\frac{z^{2}}{z^{2}+1} \\
\mathcal{L}\left(y_{2}(t)\right)=\frac{2 z^{2}}{4 z^{2}+1}
\end{array}\right.
$$

Recalling $z=\frac{1}{s}$ to obtain $\left[\frac{2}{2}\right]$ in terms of $s$

$$
\left[\frac{2}{2}\right]=\left\{\begin{array}{l}
\mathcal{L}\left(y_{1}(t)\right)=\frac{1}{s^{2}+1} \\
\mathcal{L}\left(y_{2}(t)\right)=\frac{2}{s^{2}+4}
\end{array}\right.
$$

Applying the inverse Laplace transform to the $\left[\frac{2}{2}\right]$ Padé approximant, we attain the approximate modified solution

$$
\left\{\begin{array}{c}
y_{1}(t)=\sin (t) \\
y_{2}(t)=\sin (2 t)
\end{array}\right.
$$

which agrees with the exact solution for the given problem. It is noted that the MDTS is needed just until the first $\mathcal{O}\left(t^{5}\right)$ terms to get the exact solution.

Figure 1 shows the graph of the exact solutions $y_{j}(t)$, $j=1,2$ comparing the proposed method solution with the DTM solution for Problem 3.1 

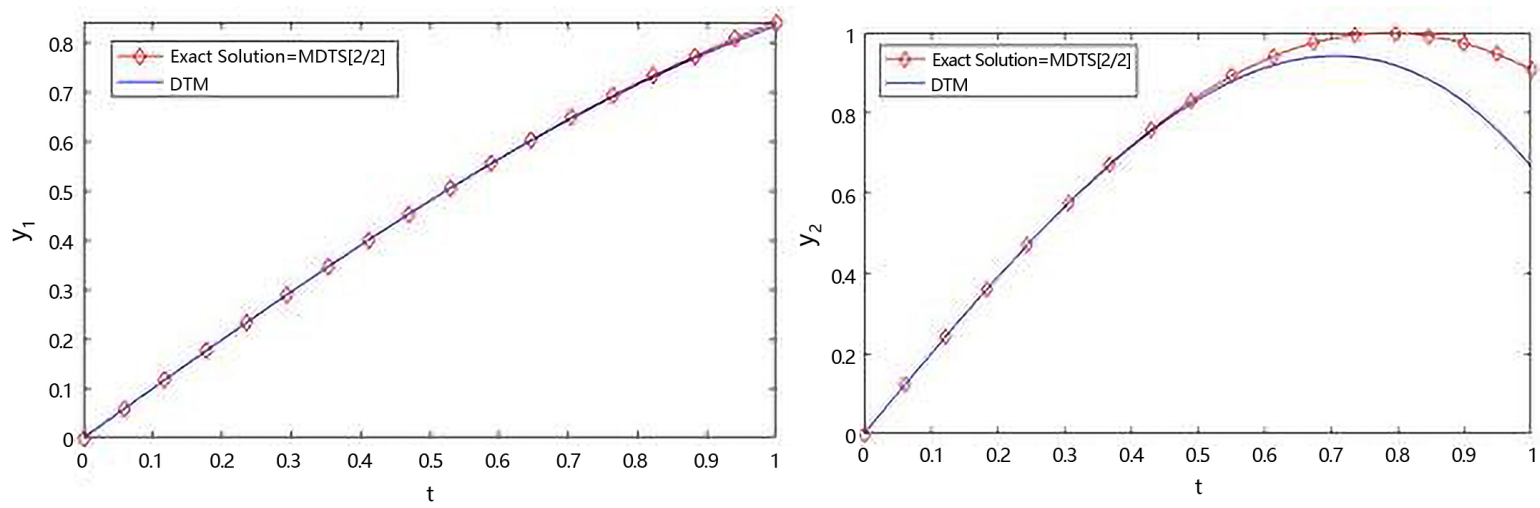

Figure 1. Comparison between the Approximated and Exact Solutions for Problem 3.1

Table 3. The values of $Y_{1}(k)$ and $Y_{2}(k)$ for Problem 3.2

\begin{tabular}{|c|c|c|c|c|c|c|}
\hline$k$ & 0 & 1 & 2 & 3 & 4 & 5 \\
\hline$Y_{1}(k)$ & 0 & 0 & 0.5 & 0 & 0 & 0 \\
\hline$Y_{2}(k)$ & 0 & 0 & 0 & 0 & -0.0416666667 & 0 \\
\hline$k$ & 6 & 7 & 8 & 9 & 10 & 11 \\
\hline$Y_{1}(k)$ & 0.0013888889 & 0 & 0 & 0 & 0.0000002756 & 0 \\
\hline$Y_{2}(k)$ & 0 & 0 & -0.0000248016 & 0 & 0 & 0 \\
\hline
\end{tabular}

Problem 3.2: Consider the second order system of DEs [16]

$$
\left\{\begin{array}{l}
y_{1}^{\prime \prime}(t)+y_{2}(t)=1 \\
y_{2}^{\prime \prime}(t)+y_{1}(t)=0
\end{array}\right.
$$

subject to

$$
\begin{cases}y_{1}(0)=0, & y_{1}{ }^{\prime}(0)=0 \\ y_{2}(0)=0, & y_{2}{ }^{\prime}(0)=0\end{cases}
$$

and exact solution

$$
\left\{\begin{array}{c}
y_{1}(t)=-\frac{\cos (t)}{2}+\frac{e^{t}}{4}+\frac{e^{-t}}{4} \\
y_{2}(t)=1-\frac{\cos (t)}{2}-\frac{e^{t}}{4}-\frac{e^{-t}}{4}
\end{array}\right.
$$

Transforming (3.8) with (3.9), to get

$$
\left\{\begin{array}{c}
(k+1)(k+2) Y_{1}(k+2)+Y_{2}(k)=\delta(k) \\
(k+1)(k+2) Y_{2}(k+2)+Y_{3}(k)=0
\end{array}\right.
$$

Thus,

$$
\left\{\begin{array}{c}
Y_{1}(k+2)=\frac{1}{(k+1)(k+2)}\left[\delta(k)-Y_{2}(k)\right] \\
Y_{1}(k+2)=-\frac{Y_{2}(k)}{(k+1)(k+2)}
\end{array}\right.
$$

and

$$
\left\{\begin{array}{l}
Y_{1}(0)=0, Y_{1}(1)=0 \\
Y_{2}(0)=0, Y_{2}(1)=0
\end{array}\right.
$$

Replacing (3.12) in (3.11), produces the values of $Y_{1}(k)$ and $Y_{2}(k)$ presented in Table 3.

Using the inverse transformation rule (2.2), we obtain an approximate solution of equation (3.8) in the form

$$
\left\{\begin{array}{c}
y_{1}(t)=0.5 t^{2}+0.0013888889 t^{6}+0.0000002756 t^{10}+\cdots \\
y_{2}(t)=-0.0416666667 t^{4}-0.0000248016 t^{8}+\cdots
\end{array}\right.
$$

The MDTS is applied to improve the efficiency of
(3.13). By taking just until $\mathcal{O}\left(t^{8}\right)$ terms from (3.13), the MDTS is executed as follows: Applying the Laplace transform to the first $\mathcal{O}\left(t^{8}\right)$ terms from (3.13), yields

$$
\left\{\begin{array}{c}
\mathcal{L}\left(y_{1}(t)\right)=\frac{1}{s^{3}}+\frac{1}{s^{7}} \\
\mathcal{L}\left(y_{2}(t)\right)=-\frac{1}{s^{5}}-\frac{1}{s^{9}}
\end{array}\right.
$$

For simplicity, let $s=\frac{1}{z}$; then

$$
\left\{\begin{array}{c}
\mathcal{L}\left(y_{1}(t)\right)=z^{3}+z^{7} \\
\mathcal{L}\left(y_{2}(t)\right)=-z^{5}-z^{9}
\end{array}\right.
$$

The Padé approximants $\left[\frac{5}{4}\right]$ gives

$$
\left[\frac{5}{4}\right]=\left\{\begin{array}{l}
\mathcal{L}\left(y_{1}(t)\right)=-\frac{z^{3}}{z^{4}-1} \\
\mathcal{L}\left(y_{2}(t)\right)=\frac{z^{5}}{z^{4}-1}
\end{array}\right.
$$

Recalling $z=\frac{1}{s}$ to obtain $\left[\frac{5}{4}\right]$ in terms of $s$

$$
\left[\frac{5}{4}\right]=\left\{\begin{array}{l}
\mathcal{L}\left(y_{1}(t)\right)=\frac{s}{s^{4}-1} \\
\mathcal{L}\left(y_{2}(t)\right)=-\frac{1}{s^{5}-s}
\end{array}\right.
$$

Employing the Laplace transform inverse to the $\left[\frac{5}{4}\right]$ Padé approximant to obtain the approximate modified solution yield

$$
\left\{\begin{array}{c}
y_{1}(t)=-\frac{\cos (t)}{2}+\frac{e^{t}}{4}+\frac{e^{-t}}{4} \\
y_{2}(t)=1-\frac{\cos (t)}{2}-\frac{e^{t}}{4}-\frac{e^{-t}}{4}
\end{array}\right.
$$

which agrees with the exact solution. It is obvious to see that the MDTS is needed just until the first $\mathcal{O}\left(t^{8}\right)$ terms to get the exact solution.

Figure 2 shows the graph of the exact solution $y_{j}(t)$, $j=1,2$ comparing the proposed MDTM solution with the DTM solution [16] for Problem 3.2. 

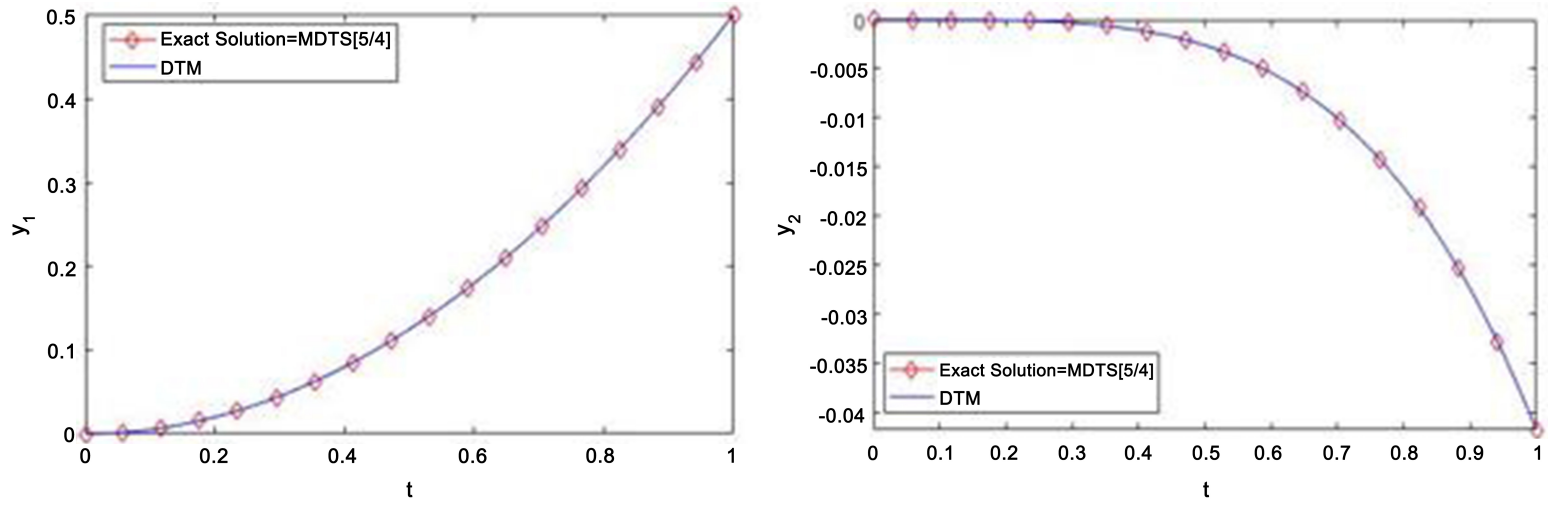

Figure 2. Comparison between the Approximated and Exact Solutions for Problem 3.2

Table 4. The values of $Y_{1}(k), Y_{2}(k)$ and $Y_{3}(k)$ for Problem 3.3

\begin{tabular}{|c|c|c|c|c|c|c|}
\hline$k$ & 0 & 1 & 2 & 3 & 4 & 5 \\
\hline$Y_{1}(k)$ & 0 & 1 & 1 & 0.5 & 0.1666666667 & 0.0416666667 \\
\hline$Y_{2}(k)$ & 1 & 2 & 1.5 & 0.6666666667 & 0.2083333333 & 0.05 \\
\hline$Y_{3}(k)$ & 2 & 3 & 2 & 0.8333333333 & 0.25 & 0.0583333333 \\
\hline
\end{tabular}

Problem 3.3: Given the third order non-linear ordinary differential equation [2]:

$$
y^{\prime \prime \prime}=\frac{1}{t} y+y^{\prime \prime}
$$

where

$$
y(0)=0, y^{\prime}(0)=1, y^{\prime \prime}(0)=2,
$$

and exact solution $y(t)=t e^{t}$.

Considering three functions, $y_{1}(t)=y(t), y_{2}(t)=$ $y^{\prime}(t)$, and $y_{3}(t)=y^{\prime \prime}(t)$, Eq.(3.16) can be transformed to the first order non-linear system of three differential equations as follows.

$$
\left\{\begin{array}{c}
y_{1}{ }^{\prime}(t)=y_{2}(t) \\
y_{2}{ }^{\prime}(t)=y_{3}(t) \\
y_{3}^{\prime}(t)=\frac{y_{1}(t)}{t}+y_{3}(t)
\end{array}\right.
$$

subject to conditions

$$
\left\{\begin{array}{l}
y_{1}(0)=0 \\
y_{2}(0)=1 \\
y_{3}(0)=2
\end{array}\right.
$$

Transforming (3.18) with (3.19), to obtain

$$
\left\{\begin{array}{c}
(k+1) Y_{1}(k+1)=Y_{2}(k) \\
(k+1) Y_{2}(k+1)=Y_{3}(k) \\
(k+1) Y_{3}(k+1)=Y_{1}(k+1)+Y_{3}(k)
\end{array},\right.
$$

Thus,

$$
\left\{\begin{array}{c}
Y_{1}(k+1)=\frac{Y_{2}(k)}{k+1} \\
Y_{2}(k+1)=\frac{Y_{3}(k)}{k+1} \\
Y_{3}(k+1)=\frac{1}{k+1}\left[Y_{1}(k+1)+Y_{3}(k)\right]
\end{array}\right.
$$

and

$$
\left\{\begin{array}{l}
Y_{1}(0)=0 \\
Y_{2}(0)=1 \\
Y_{3}(0)=2
\end{array}\right.
$$

Replacing (3.22) in (3.21), produces the values of $Y_{1}(k), Y_{2}(k)$ and $Y_{3}(k)$ which are presented in Table 4.

Applying rule in (2.2), we obtain an approximate solution of equation (3.18) in the form

$\left\{\begin{array}{c}y_{1}(t)=t+t^{2}+0.5 t^{3}+0.1666666667 t^{4}+0.0416666667 t^{5}+\cdots \\ y_{2}(t)=1+2 t+1.5 t^{2}+0.6666666667 t^{3}+0.2083333333 t^{4}+0.05 t^{5}+\cdots, \\ y_{3}(t)=2+3 t+2 t^{2}+0.8333333333 t^{3}+0.25 t^{4}+0.0583333333 t^{5}+\cdots\end{array}\right.$

To improve the efficiency of (3.23), and to prove the efficiency of MDTS, we proceed as follows. By taking just $\mathcal{O}\left(t^{5}\right)$ terms from (3.23), the MDTS is employed as follows: Applying the Laplace transform to the first $\mathcal{O}\left(t^{5}\right)$ terms from (5.68), yields

$$
\left\{\begin{array}{c}
\mathcal{L}\left(y_{1}(t)\right)=\frac{1}{s^{2}}+\frac{2}{s^{3}}+\frac{3}{s^{4}}+\frac{4}{s^{5}}+\frac{5}{s^{6}} \\
\mathcal{L}\left(y_{2}(t)\right)=\frac{1}{s}+\frac{2}{s^{2}}+\frac{3}{s^{3}}+\frac{4}{s^{4}}+\frac{5}{s^{5}}+\frac{6}{s^{6}} \\
\mathcal{L}\left(y_{3}(t)\right)=\frac{2}{s}+\frac{3}{s^{2}}+\frac{4}{s^{3}}+\frac{5}{s^{4}}+\frac{6}{s^{5}}+\frac{7}{s^{6}}
\end{array}\right.
$$

For simplicity, let $s=\frac{1}{z}$; then

$$
\left\{\begin{array}{c}
\mathcal{L}\left(y_{1}(t)\right)=z^{2}+2 z^{3}+3 z^{4}+4 z^{5}+5 z^{6} \\
\mathcal{L}\left(y_{2}(t)\right)=z+2 z^{2}+3 z^{3}+4 z^{4}+5 z^{5}+6 z^{6} \\
\mathcal{L}\left(y_{3}(t)\right)=2 z+3 z^{2}+4 z^{3}+5 z^{4}+6 z^{5}+7 z^{6}
\end{array}\right.
$$

The Padé approximants $\left[\frac{3}{3}\right]$ gives 


$$
\left[\frac{3}{3}\right]=\left\{\begin{array}{l}
\mathcal{L}\left(y_{1}(t)\right)=\frac{z^{2}}{z^{2}-2 z+1} \\
\mathcal{L}\left(y_{2}(t)\right)=\frac{z}{z^{2}-2 z+1} \\
\mathcal{L}\left(y_{3}(t)\right)=\frac{-z^{2}+2 z}{z^{2}-2 z+1}
\end{array}\right.
$$

Recalling $z=\frac{1}{s}$ to obtain $\left[\frac{3}{3}\right]$ in terms of $s$

$$
\left[\begin{array}{l}
3 \\
3
\end{array}\right]=\left\{\begin{array}{l}
\mathcal{L}\left(y_{1}(t)\right)=\frac{1}{s^{2}-2 s+1} \\
\mathcal{L}\left(y_{2}(t)\right)=\frac{s}{s^{2}-2 s+1} \\
\mathcal{L}\left(y_{3}(t)\right)=\frac{2 s-1}{s^{2}-2 s+1}
\end{array}\right.
$$

Employing Laplace transform inverse to the $\left[\frac{3}{3}\right]$ Padé approximant, we obtain the approximate modified solution

$$
\left\{\begin{array}{c}
y_{1}(t)=t e^{t} \\
y_{2}(t)=e^{t}+t e^{t} \\
y_{3}(t)=2 e^{t}+t e^{t}
\end{array}\right.
$$

Therefore,

$$
y(t)=y_{1}(t)=t e^{t}
$$

which agrees with the exact solution for this Example.

Problem 3.4: Given the third order Euler-Lagrange equation [17]:

$$
y^{\prime \prime \prime}=-\frac{1}{x^{2}} y^{\prime}+\frac{1}{x^{3}} y
$$

with

$$
y(1)=1, y^{\prime}(1)=2, y^{\prime \prime}(1)=3,
$$

and exact solution $y(x)=x\left(1+\ln (x)+(\ln (x))^{2}\right)$.

To overcome the singularity difficulties met at $x=0$ and to achieve the proposed objective, we need to transform the problem as follows

$$
t=\ln (x), \quad x=e^{t},
$$

so that

$$
\frac{d y}{d x}=\frac{1}{x} \frac{d y}{d t}
$$

$$
\begin{gathered}
\frac{d^{2} y}{d x^{2}}=\frac{1}{x^{2}} \frac{d^{2} y}{d t^{2}}-\frac{1}{x^{2}} \frac{d y}{d t} \\
\frac{d^{3} y}{d x^{3}}=\frac{1}{x^{3}} \frac{d^{3} y}{d t^{3}}-\frac{3}{x^{3}} \frac{d^{2} y}{d t^{2}}+\frac{2}{x^{3}} \frac{d y}{d t}
\end{gathered}
$$

Subsisting (3.29), (3.30), and (3.31) in (3.26), we obtain

$$
\frac{d^{3} y}{d t^{3}}-3 \frac{d^{2} y}{d t^{2}}+3 \frac{d y}{d t}-y(t)=0
$$

with initial conditions

$$
y(t=0)=1, y^{\prime}(t=0)=2, y^{\prime \prime}(t=0)=5,
$$

Considering three functions, $y_{1}(t)=y(t), y_{2}(t)=\frac{d y}{d t}$, and $y_{3}(t)=\frac{d^{2} y}{d t^{2}}$, Eq.(3.32) can be transformed into a first order non-linear system of three differential equations as follows.

$$
\left\{\begin{array}{c}
y_{1}{ }^{\prime}(t)=y_{2}(t) \\
y_{2}{ }^{\prime}(t)=y_{3}(t) \\
y_{3}{ }^{\prime}(t)=y_{1}(t)-3 y_{2}(t)+3 y_{3}(t)
\end{array},\right.
$$

subject to conditions

$$
\left\{\begin{array}{l}
y_{1}(0)=1 \\
y_{2}(0)=2 \\
y_{3}(0)=5
\end{array}\right.
$$

Transforming Eq. (3.34) with the initial conditions Eq. (3.35), to obtain

$$
\left\{\begin{array}{c}
(k+1) Y_{1}(k+1)=Y_{2}(k) \\
(k+1) Y_{2}(k+1)=Y_{3}(k) \\
(k+1) Y_{3}(k+1)=Y_{1}(k)-3 Y_{2}(k)+3 Y_{3}(k)
\end{array},\right.
$$

Thus,

$$
\left\{\begin{array}{c}
Y_{1}(k+1)=\frac{Y_{2}(k)}{k+1} \\
Y_{2}(k+1)=\frac{Y_{3}(k)}{k+1} \\
Y_{3}(k+1)=\frac{1}{k+1}\left[Y_{1}(k)-3 Y_{2}(k)+3 Y_{3}(k)\right]
\end{array},\right.
$$

and

$$
\left\{\begin{array}{l}
Y_{1}(0)=1 \\
Y_{2}(0)=2 \\
Y_{3}(0)=5
\end{array}\right.
$$

Replacing (3.38) in (3.37), produce the values of $Y_{1}(k), Y_{2}(k)$ and $Y_{3}(k)$ which are shown in Table 5.

Table 5. The values of $Y_{1}(k), Y_{2}(k)$ and $Y_{3}(k)$ for Problem 3.4

\begin{tabular}{|c|c|c|c|c|c|c|}
\hline$k$ & 0 & 1 & 2 & 3 & 4 & 5 \\
\hline$Y_{1}(k)$ & 1 & 2 & 2.5 & 1.6666666667 & 0.7083333333 & 0.2166666667 \\
\hline$Y_{2}(k)$ & 2 & 5 & 5 & 2.8333333333 & 1.0833333333 & 0.3083333333 \\
\hline$Y_{3}(k)$ & 5 & 10 & 8.5 & 4.3333333333 & 1.5416666667 & 0.4166666667 \\
\hline
\end{tabular}


Applying the rule in (2.2), we obtain an approximate solution of equation (3.34) in the form

$\left\{y_{1}(t)=1+2 t+2.5 t^{2}+1.66666666667 t^{3}+0.7083333333 t^{4}+0.2166666667 t^{5}+\cdots\right.$ $\left\{\begin{array}{l}y_{2}(t)=2+5 t+5 t^{2}+2.8333333333 t^{3}+1.0833333333 t^{4}+0.30833333333 t^{5}+\cdots \\ y_{3}(t)=5+10 t+8.5 t^{2}+43333333333 t^{3}+1.5416666667 t^{4}+0.416666666 t^{5}+\cdots\end{array}\right.$ $\left\{\begin{array}{c}y_{3}(t)=5+10 t+8.5 t^{2}+4.33333333333 t^{3}+1.5416666667 t^{4}+0.4166666667 t^{5}+\cdots \\ y^{2}\end{array}\right.$

To enhance the efficiency of (3.39), and to prove the efficiency for the MDTS, we proceed as follows. By taking just $\mathcal{O}\left(t^{5}\right)$ terms from (3.39), the MDTS is applied as follows: Applying the Laplace transform to the first $\mathcal{O}\left(t^{5}\right)$ terms from (3.39), yields

$$
\left\{\begin{array}{l}
\mathcal{L}\left(y_{1}(t)\right)=\frac{1}{s}+\frac{2}{s^{2}}+\frac{5}{s^{3}}+\frac{10}{s^{4}}+\frac{17}{s^{5}}+\frac{26}{s^{6}} \\
\mathcal{L}\left(y_{2}(t)\right)=\frac{2}{s}+\frac{5}{s^{2}}+\frac{10}{s^{3}}+\frac{17}{s^{4}}+\frac{26}{s^{5}}+\frac{37}{s^{6}} \\
\mathcal{L}\left(y_{3}(t)\right)=\frac{5}{s}+\frac{10}{s^{2}}+\frac{17}{s^{3}}+\frac{26}{s^{4}}+\frac{37}{s^{5}}+\frac{50}{s^{6}}
\end{array}\right.
$$

For simplicity, let $s=\frac{1}{z}$; then

$$
\left\{\begin{array}{c}
\mathcal{L}\left(y_{1}(t)\right)=z+2 z^{2}+5 z^{3}+10 z^{4}+17 z^{5}+26 z^{6} \\
\mathcal{L}\left(y_{2}(t)\right)=2 z+5 z^{2}+10 z^{3}+17 z^{4}+26 z^{5}+37 z^{6} \\
\mathcal{L}\left(y_{3}(t)\right)=5 z+10 z^{2}+17 z^{3}+26 z^{4}+37 z^{5}+50 z^{6}
\end{array}\right.
$$

The Padé approximants $\left[\frac{3}{3}\right]$ gives

$$
\left[\frac{3}{3}\right]=\left\{\begin{array}{l}
\mathcal{L}\left(y_{1}(t)\right)=\frac{-2 z^{3}+z^{2}-z}{z^{3}-3 z^{2}+3 z-1} \\
\mathcal{L}\left(y_{2}(t)\right)=\frac{-z^{3}+z^{2}-2 z}{z^{3}-3 z^{2}+3 z-1} \\
\mathcal{L}\left(y_{3}(t)\right)=\frac{-2 z^{3}+5 z^{2}-5 z}{z^{3}-3 z^{2}+3 z-1}
\end{array}\right.
$$

Recalling $z=\frac{1}{s}$ to obtain $\left[\frac{3}{3}\right]$ in terms of $s$

$$
\left[\begin{array}{l}
\frac{3}{3}
\end{array}\right]=\left\{\begin{array}{l}
\mathcal{L}\left(y_{1}(t)\right)=\frac{-s^{2}+s-2}{-s^{3}+3 s^{2}-3 s+1} \\
\mathcal{L}\left(y_{2}(t)\right)=\frac{-2 s^{2}+s-1}{-s^{3}+3 s^{2}-3 s+1} \\
\mathcal{L}\left(y_{3}(t)\right)=\frac{-5 s^{2}+5 s-2}{-s^{3}+3 s^{2}-3 s+1}
\end{array}\right.
$$

Applying Laplace transform inverse to the $\left[\frac{3}{3}\right]$ Padé approximant to attain the approximate modified solution

$$
\left\{\begin{array}{c}
y_{1}(t)=e^{t}\left(1+t+t^{2}\right) \\
y_{2}(t)=e^{t}\left(2+3 t+t^{2}\right) \\
y_{3}(t)=e^{t}\left(5+5 t+t^{2}\right)
\end{array}\right.
$$

Therefore,

$$
y(t)=y_{1}(t)=e^{t}\left(1+t+t^{2}\right)
$$

Subsisting $t=\ln (x)$, to get

$$
y(x)=x\left(1+\ln (x)+(\ln (x))^{2}\right)
$$

which is in excellent agreement with the exact solution for this Examples

\section{Conclusions and Discussion}

The MDTS is a competitive and robust method for obtaining the approximate solutions of second order systems of DEs and higher order DEs. All the preliminary results obtained had shown that the proposed scheme is in excellent agreement with all results of the exact solution. These problems illustrate that the MDTM has significantly improved the rate of convergence of the truncated solution of the series obtained by DTM, and frequently produce the true analytic solution. Some performance comparison graphs are also presented to illustrate the efficiency of the proposed method. The overall results show that the proposed scheme is promising and applicable in other fields.

\section{REFERENCES}

[1] Mirzaee, F., Differential transform method for solving linear and nonlinear systems of ordinary differential equations. Applied Mathematical Sciences, 2011. 5(70): p. 3465-3472.

[2] Biazar, J., E. Babolian, and R. Islam, Solution of the system of ordinary differential equations by Adomian decomposition method. Applied Mathematics and Computation, 2004. 147(3): p. 713-719.

[3] Zhou, Differential transformation and its applications for electrical circuits. 1986, Huazhong University Press, Wuhan, China.

[4] Chen, C.-L. and L. Sy-Hong, Application of Taylor transformation to nonlinear predictive control problem. Applied mathematical modelling, 1996. 20(9): p. 699-710.

[5] Ayaz, F., Solutions of the system of differential equations by differential transform method. Applied Mathematics and Computation, 2004. 147(2): p. 547-567.

[6] Odibat, Z.M., Differential transform method for solving Volterra integral equation with separable kernels. Mathematical and Computer Modelling, 2008. 48(7-8): p. 1144-1149.

[7] Momani, S. and V.S. Ertürk, Solutions of non-linear oscillators by the modified differential transform method. Computers \& Mathematics with Applications, 2008. 55(4): p. $833-842$.

[8] Al-Ahmad, S., et al., On New Properties of Differential Transform via Difference Equations. International Journal of Engineering \& Technology, 2018. 7(3.28): p. 321-324.

[9] Ibis, B., Approximate analytical solutions for nonlinear Emden-Fowler type equations by differential transform method. arXiv preprint arXiv:1211.3521, 2012. 
[10] Ali, A.H., Applications of Differential Transform Method to Initial Value Problems. American Journal of Engineering Research 2017. 6(12): p. 365-371.

[11] Jameel, A., et al., Differential Transformation Method for Solving High Order Fuzzy Initial Value Problems. Italian Journal of Pure and Applied Mathematics, 2018. 39: p. 194-208.

[12] Kanth, A.R. and K. Aruna, Differential transform method for solving linear and non-linear systems of partial differential equations. Physics Letters A, 2008. 372(46): p. 6896-6898.

[13] Al-Ahmad, S., M. Mamat, and R. AlAhmad, Finding Differential Transform Using Difference Equations. International Journal of Applied Mathematics, 2020. 50(1).

[14] Bender, C.M. and S.A. Orszag, Advanced mathematical methods for scientists and engineers I: Asymptotic methods and perturbation theory. 2013: Springer Science \& Business Media.

[15] Baker, G.A., Essentials of Padé approximants. 1975: academic press.

[16] Thongmoon, M. and S. Pusjuso, The numerical solutions of differential transform method and the Laplace transform method for a system of differential equations. Nonlinear Analysis: Hybrid Systems, 2010. 4(3): p. 425-431.

[17] Biazar, J. and H. Ghazvini, He’s variational iteration method for solving linear and non-linear systems of ordinary differential equations. Applied Mathematics and Computation, 2007. 191(1): p. 287-297.

[18] Al-Ahmad, S., Sulaiman, I.M., Mamat, M., and Ghazali, P.L. Modified Differential Transform Scheme for Solving Systems of First Order Ordinary Differential Equations. Journal of Mathematics and Computer Science, 2021, 22: p. 73-84. 